\title{
A non-interactive deniable authentication scheme in the standard model
}

\author{
Bin Wang ,Qing Zhao and Ke Dai \\ Information Engineering College of Yangzhou University \\ No.196 West HuaYang Road, Yangzhou City, Jiangsu Province, P.R.China \\ E-mail: jxbin76@yahoo.cn
}

\begin{abstract}
Deniable authentication protocols enable a sender to authenticate a message to a receiver such that the receiver is unable to prove the identity of the sender to a third party. In contrast to interactive schemes, non-interactive deniable authentication schemes improve communication efficiency. Currently, several non-interactive deniable authentication schemes have been proposed with provable security in the random oracle model. In this paper, we study the problem of constructing non-interactive deniable authentication scheme secure in the standard model without bilinear groups. An efficient non-interactive deniable authentication scheme is presented by combining the Diffie-Hellman key exchange protocol with authenticated encryption schemes. We prove the security of our scheme by sequences of games and show that the computational cost of our construction can be dramatically reduced by applying pre-computation technique.
\end{abstract}

Keywords: Deniable authentication; Authenticated encryption; Diffie-Hellman key exchange; The standard model; Sequences of games

\section{Introduction}

One of the main fields of interest in cryptography is the design and analysis of authentication schemes. Deniable authentication schemes enable a sender to authenticate a message to a receiver such that the specified receiver is unable to prove to a third party that the message is authenticated by the sender. Deniable authentication schemes are useful in electronic voting systems and secure negotiation over the Internet where the sender's identity should only be revealed to the specified receiver.

The concept of deniable authentication was initially developed by Dwork et al. [10] based on concurrent zero-knowledge proof. But their scheme required a timing constraint on 
the network and the proof of knowledge was rather time-consuming. Another deniable authentication protocol was developed independently by Aumann and Rabin [1] under the factoring assumption. Later, Deng [9] proposed two deniable authentication schemes based on the factoring problem and the discrete logarithm problem respectively. Fan [11] proposed a simple deniable authentication protocol based on the Diffie-Hellman key exchange protocol. But the schemes $[9,11]$ did not provide formal analysis and were broken or improved in [23,25]. Raimondo et al. [19] considered new approaches for deniable authentication while providing guaranteed provable-security. They [19] extended the ideas from authenticated key exchange protocols [3] to the setting of deniable authentication protocols.

However, all of the above-mentioned deniable authentication protocols are interactive protocols. To reduce the communication cost, several non-interactive deniable authentication schemes have been proposed [14-16, 20]. Nevertheless, these non-interactive schemes did not present a rigorous security model to specify adversary's capabilities and goal. So they can only provide a weak security guarantee. For example, an improved scheme was proposed in [17] to correct a security flaw in [16]. Consequently, to analyze the security of non-interactive deniable authentication schemes, it is important to base the security proof on a formalized security model.

Later, Wang et al. [22] presented a formal model for deniable authentication based on the security model for traditional authentication schemes [5]. They also designed a non-interactive deniable authentication scheme based on designated verifier proofs [13] and proved their scheme to be secure under the DDH assumption. Recently, Youn et al. [24] presented a more efficient non-interactive deniable authentication scheme based on trapdoor commitment, which is proved to be secure under the security model in [22].

However, [22,24] proved security in the random oracle model. In cryptography, the random oracle model is a useful tool to prove the security of cryptographic schemes. However, such kind of security proof relies on the existence of random functions (that is, cryptographic hash functions replaced by elaborately designed random oracles). There are examples of schemes [2,7], which are secure in the random oracle model but are vulnerable to attacks when the random oracle is replaced by cryptographic hash functions. So it is desirable to design cryptographic schemes in the standard model, in which the adversary is only limited 
by the amount of time and computational power available.

Susilo et al. [21] provided generic constructions for non-interactive deniable ring authentication via ring signature and chameleon hash function. Strictly speaking, a 2-user ring signature schemes is sufficient for their construction to yield a deniable authentication scheme. However, the existing 2-user ring signature schemes in the standard model are built upon bilinear groups such that they are rather costly to be implemented. For instance, the 2-user ring signature schemes mentioned in [6] and the scheme in [8] require at least 3 pairing operations for verification. As pairing operations require more computational cost than exponentiation operations, it is natural to ask whether we can obtain a more efficient deniable authentication scheme such that the underlying primitives can be instantiated without relying on random oracle as well as bilinear groups. In addition, the use of chameleon hash function in their construction may induce additional computational (e.g., exponentiation operations and an implicit mapping from the output of chameleon hash function to the message domain of ring signature scheme) and communication cost(e.g., the randomness used by chameleon hash function should be included).

Hence the goal of this paper is to design efficient non-interactive deniable authentication schemes secure in the standard model that can be instantiated without bilinear groups. At first, we provide a generic construction for deniable authentication such that the deniability is based on the Diffie-Hellman key exchange protocol. Subsequently, we prove that our construction is secure against impersonation attack under the security model in [22] in the standard model by sequences of games. To prove the unforgeability of our construction, we make use of the notion of integrity of plaintexts from authenticated symmetric encryption. Finally we compare the efficiency with other non-interactive deniable authentication schemes with provable-security in the random oracle model. The result shows that the performance of our construction is comparable to those non-interactive schemes in terms of the computational cost.

\section{Preliminaries}

We denote by $k$ a security parameter. If $A$ is a randomized algorithm, then 
$y \leftarrow A\left(x_{1}, x_{2}, \cdots ; r\right)$ means that $A$ has input $x_{1}, x_{2}, \cdots$ and random coins $r$, and the output of $A$ is assigned to $y$. We use the notation $x \leftarrow{ }_{R} S$ to mean "the element $x$ is chosen with uniform probability from the set $S$ ”.

\subsection{DDH assumption}

Let $\mathbb{G}=\left\{G_{k}\right\}_{k}$ be a family of groups where $G_{k}$ has prime order $2^{k-1}<q_{k}<2^{k}$.

Given random generators $g_{1}, g_{2}$ of $G_{k}$, consider the following distributions:

$$
\begin{aligned}
& D H_{k}=\left\{\left(g_{1}, g_{2}, g_{1}^{r}, g_{2}^{r}\right) \mid r \leftarrow_{R} Z_{q_{k}}\right\} \\
& \text { Rand }_{k}=\left\{\left(g_{1}, g_{2}, g_{1}^{r_{1}}, g_{2}^{r_{2}}\right) \mid r_{1}, r_{2} \leftarrow_{R} Z_{q_{k}}\right\}
\end{aligned}
$$

For an adversary $A$, his distinguishing advantage is defined as follows:

$$
A d v_{A, G_{k}}^{D D H}(k)=\left|\operatorname{Pr}_{\tau \leftarrow{ }_{R} D H_{k}}[A(\tau)=1]-\operatorname{Pr}_{\tau \leftarrow{ }_{R} \text { Rand }_{k}}[A(\tau)=1]\right| \text {, where } \tau \text { is of the form }
$$

$\left(g_{1}, g_{2}, u_{1}, u_{2}\right)$.

We say that DDH assumption hold over the group family $\mathbb{G}=\left\{G_{k}\right\}_{k}$ if for all PPT (probabilistic polynomial-time) adversary $A, A d v_{A, G_{k}}^{D D H}(k)$ is negligible.

\subsection{Key Derivation Function}

A key derivation function $K D F$ is defined as follows:

$K D F: D o m \rightarrow\{0,1\}^{k}$.

For an adversary $A$, his distinguishing advantage is defined as follows:

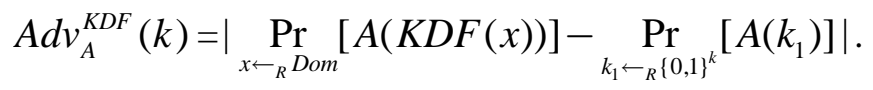

We say that $K D F$ is a secure key derivation function if for all PPT adversary $A$, $A d v_{A}^{K D F}(k)$ is negligible.

\subsection{INT-PTXT secure symmetric encryption}

Let $\operatorname{SKE}=(E, D)$ be a symmetric encryption scheme. A game $\operatorname{Exp}_{S E}^{P T X T}(k)$ [4] between an adversary $A$ and a game challenger $\mathrm{S}$ is defined as follows:

(1) The challenger $S$ picks an encryption key $e k \leftarrow_{R}\{0,1\}^{k}$ and a set $E Q$ which is initialized to empty. 
(2) An encryption query $M_{i}$ issued by the adversary $A$ is handled as follows:

$\mathrm{S}$ computes $C_{i} \leftarrow E_{e k}\left(M_{i}\right)$ and sets $E Q \leftarrow E Q \bigcup\left\{M_{i}\right\}$. Then $C_{i}$ is returned to

A.

(3) Finally $A$ outputs a ciphertext $C^{*}$.

Let $M^{*}=D_{e k}\left(C^{*}\right)$. If $M^{*} \neq \perp$ and $M^{*} \notin E Q$, then $A$ wins the game.

The advantage $A d v_{A}^{P T X T}(k)$ of the adversary $A$ in this game is defined to be the probability that $A$ wins the game. A symmetric encryption scheme provides integrity of plaintexts (INT-PTXT secure) if for all PPT adversary $A$, the advantage $A d v_{A}^{\text {PTXT }}(k)$ is negligible. Bellare [4] demonstrated that the property of integrity of plaintexts can be achieved by applying the Encrypt-and-MAC composition method to a symmetric encryption scheme and a message authentication scheme.

\subsection{One-time secure signature}

A signature scheme consists of the following algorithms:

(1) Key generation Gen: takes as input the security parameter $k$ and outputs a public key $p k$ and a matching secret key sk.

(2) Signing Sign: takes the secret key sk and a message $M$ as input and outputs a signature by computing $\sigma \leftarrow \operatorname{Sign}(s k, M)$.

(3) Verification Vrfy : takes as input a public key $p k$, a message $M$ and a signature $\sigma$. $\operatorname{Vrfy}(p k, M, \sigma)$ outputs 1 if $\sigma$ is valid and 0 otherwise.

Then we consider the following game $\operatorname{Exp}_{\text {sig }}^{1 C M A}(k)$ between an adversary $A$ and a challenger $\mathrm{S}$ :

(1) S runs $\operatorname{Gen}\left(1^{k}\right)$ to obtain the key pair $p k, s k$.

(2) $A$ is given $p k$ and is allowed to issue a signature query $M$ only once. Then $\mathrm{S}$ returns $\sigma \leftarrow \operatorname{Sign}(s k, M)$ to $A$. 
(3) $A$ outputs $\left(M^{*}, \sigma^{*}\right)$.

$A$ wins the game if $\operatorname{Vrfy}\left(p k, M^{*}, \sigma^{*}\right)=1 \wedge\left(M^{*} \neq M\right)$.

A signature scheme is existentially unforgeable under a one-time chosen message attack if for all PPT adversary $A$, the success probability $A d v_{A}^{1 C M A}(k)$ of $A$ in the above game is negligible.

\subsection{Groth's one-time secure signature}

Given a group $G$ of order $q$ with generator $g$ and a hash function $H:\{0,1\}^{*} \rightarrow Z_{q}$, we now describe a one-time signature scheme from Groth [10] whose security is proved in the standard model based on the discrete logarithm problem and collision-resistance of $H$.

(1) Key generation : Picks $x, y \in Z_{q}^{*}$ and sets $f=g^{x}$ and $h=g^{y}$. Then picks $r, s \in Z_{q}$ and sets $c=f^{r} h^{s}$. The public key is $p k=(f, h, c)$ and the secret key is $s k=(p k,(x, y, r, s))$.

(2) Signing: To sign a message $m \in\{0,1\}^{*}$, picks $t \leftarrow_{R} Z_{q}$. The signature is $\sigma=(t,(x(r-t)+y \cdot s-H(m)) / y)$.

(3) Verification: To verify the signature $\sigma=(t, w)$, checks that $c=g^{H(m)} f^{t} h^{w}$.

\section{Deniable authentication schemes}

\subsection{Syntax of deniable authentication schemes}

A non-interactive deniable authentication scheme consists of the following algorithms [22]:

(1) Setup: Given a security parameter $k$, generates common system parameters cps.

(2) KeyGen: Given cps, generates a public key $p k$ and a matching secret key sk .

(3) $\mathrm{P}$ : Given a message $M$, the prover runs $\mathrm{P}\left(p k_{V}, s k_{P}, M ; \rho\right)$ to generate an authenticator Authen, where $p k_{V}$ is the public key of the verifier, $s k_{P}$ is the secret key of the prover and $\rho$ is the randomness. Then the prover sends $M \|$ Authen to the verifier. 
The conversation transcript $C$ is defined to be $M \|$ Authen .

(4) V: Given the transcript $C$, the verifier runs $V\left(M, A u t h e n, s k_{V}, p k_{P}\right)$ to output a decision bit $d \in\{0,1\}$. $d=1$ means that the verifier accepts.

Correctness: For all cps $\leftarrow \operatorname{Setup}\left(1^{k}\right), \quad(p k, s k) \leftarrow \operatorname{KeyGen}(\mathrm{cps})$, we require perfect consistency, meaning that $\operatorname{Pr}\left[d=1:\left[\begin{array}{l}\text { Authen } \leftarrow P\left(p k_{V}, s k_{P}, M ; \rho\right) \\ d \leftarrow V\left(M, \text { Authen, } s k_{V}, p k_{P}\right)\end{array}\right]\right]=1$.

(5) Sim: Given the prover's public key $p k_{P}$ and the verifier's secret key $s k_{V}$, the simulation algorithm Sim generates a simulated authenticator $\overline{\text { Authen }} \leftarrow \operatorname{Sim}\left(p k_{P}, s k_{V}, M ; \bar{\rho}\right)$.

\subsection{Security model for deniable authentication schemes}

\subsubsection{Unforgeability}

Let NDI = (Setup, KeyGen,P,V,Sim) be a non-interactive deniable authentication scheme. Consider the following game $\operatorname{Exp}_{\mathrm{NDI}, \mathrm{A}}^{\mathrm{imp}}(k)$ between an adversary $A$ and a game challenger $\mathrm{S}[22]$ :

Stage 1: The challenger $S$ runs $\operatorname{cps} \leftarrow \operatorname{Setup}\left(1^{k}\right)$, and KeyGen(cps) to obtain the prover and verifier key pairs $\left(p k_{P}, s k_{P}\right),\left(p k_{V}, s k_{V}\right)$ respectively. An empty set Res is also created, which is used to store Conv queries issued by the adversary. Then $A$ is provided with the public keys $p k_{P}, p k_{V}$.

Stage 2: The challenger $\mathrm{S}$ answers each Conv query issued by $A$ as follows:

(1) Given a message $M$ chosen by $A$, S sets the state of the prover algorithm to $S t_{P}=\left(p k_{V}, s k_{P} ; \rho\right)$, where $\rho$ denotes fresh random coins chosen by $\mathrm{S}$. Then $\mathrm{S}$ provides $A$ with Authen $\leftarrow P\left(M, S t_{P}\right)$ and sets $\operatorname{Res} \leftarrow \operatorname{Res} \bigcup\{M\}$.

Output: Eventually, $A$ outputs $S t_{A}$, which represents knowledge gained by $A$ during stage 2. If the following conditions hold, the output of the game is set to 1 to indicate that $A$ wins the game and 0 otherwise: 
(1) $\left[\begin{array}{l}\left(M^{*}, \text { Authen }^{*}\right) \leftarrow A\left(S t_{A}\right) \\ d^{*} \leftarrow V\left(M^{*}, \text { Authen }^{*}, s k_{V}, p k_{P}\right)\end{array}\right]$, and

(2) $d^{*}=1, M^{*} \notin \operatorname{Res}$.

, where $\left(M^{*}\right.$, Authen $\left.{ }^{*}\right)$ denotes the final output of the adversary $A$.

The advantage of $A$ in this game is defined as $\operatorname{Adv}_{\mathrm{NDI}, \mathrm{A}}^{\mathrm{imp}}(k)=\operatorname{Pr}\left[\operatorname{Exp}_{\mathrm{NDI}, \mathrm{A}}^{\mathrm{imp}}(k)=1\right]$. NDI is secure against impersonation attack if $\operatorname{Adv}_{\mathrm{NDI}, \mathrm{A}}^{\mathrm{imp}}(k)$ is negligible for every PPT adversary $A$.

\subsubsection{Deniability}

Consider the following game $\operatorname{Exp}_{\mathrm{NDI}, \mathrm{D}}^{\mathrm{Den}}(k)$ between a distinguisher $D$ and a game challenger $S$ [22].

Stage 1: The challenger $S$ runs cps $\leftarrow \operatorname{Setup}\left(1^{k}\right)$, and KeyGen(cps) to obtain the key pairs $\left(p k_{p}, s k_{P}\right),\left(p k_{V}, s k_{V}\right)$. Two empty sets Res and $\overline{\operatorname{Res}}$ are created. Then the distinguisher $D$ is provided with the public keys $p k_{P}, p k_{V}$.

Stage 2: The distinguisher $D$ makes the following queries:

(1) Conv queries: Given a message $M$ chosen by $D$, S sets the state of the prover algorithm to $S t_{P}=\left(p k_{V}, s k_{P} ; \rho\right)$, where $\rho$ denotes fresh random coins chosen by S. Then $\mathrm{S}$ provides $D$ with Authen $\leftarrow P\left(M, S t_{P}\right)$ and sets $\operatorname{Res} \leftarrow \operatorname{Res} \bigcup\{M\}$.

(2) $\overline{\text { Conv }}$ queries: Given a message $M$ chosen by $D, S$ sets the input of the simulation algorithm $\operatorname{Sim}$ to $S t=\left(p k_{P}, s k_{V} ; \rho\right)$, where $\rho$ denotes fresh random coins chosen by $\mathrm{S}$. Then $\mathrm{S}$ provides $\mathrm{D}$ with $\overline{\operatorname{Authen}} \leftarrow \operatorname{Sim}(M, S t)$ and sets $\overline{\operatorname{Res}} \leftarrow \overline{\operatorname{Res}} \bigcup\{M\}$

Challenge: Once $D$ decides that Stage 2 is over, $D$ picks a message $M^{*}$ such that $M^{*}$ has not been submitted as one of the Conv queries or $\overline{\operatorname{Conv}}$ queries. Then the 
challenger $S$ picks a random bit $b \in\{0,1\}$. If $b=0, S$ generates a real transcript $C$ and returns $C$ to $D$. Otherwise, $S$ generates a simulated transcript $\bar{C}$ and returns $\bar{C}$ to $D$.

Guess: Finally, $D$ outputs a bit $b^{\prime}$. If $b^{\prime}=b$, the output of the game is set to 1 to indicate that $D$ wins the game and 0 otherwise.

The advantage of $D$ in this game is defined as $\operatorname{Adv}_{\mathrm{NDI}, \mathrm{D}}^{\mathrm{Den}}(k)=\operatorname{Pr}\left[\operatorname{Exp}_{\mathrm{NDI}, \mathrm{D}}^{\mathrm{Den}}(k)=1\right]$. NDI is deniable if $\operatorname{Adv}_{\mathrm{NDI}, \mathrm{D}}^{\mathrm{Den}}(k)$ is negligible for every PPT distinguisher $D$.

\section{Our scheme}

Our scheme consists of the following algorithms:

(1) Setup: Let $G$ be a multiplicative cyclic group generated by $g$ with prime order $q, \log _{2} q=k$, where $k$ is a security parameter. Then chooses a key derivation function $K D F: G \rightarrow\{0,1\}^{k}$, a symmetric encryption scheme $S K E=(E, D)$ and a one-time secure signature scheme (Gen, Sign, Vrfy).

(2) KeyGen: Picks $x_{U} \leftarrow{ }_{R} Z_{q}$. The public key $p k_{U}$ of a user $U$ is $g^{x_{U}}$ and the secret key is $x_{U}$.

(3) P: Given a message $M$ and the public key $g^{x_{V}}$ of the verifier $V U$, the prover $P U$ proceeds as follows:

$$
\begin{aligned}
& v k=\left(g^{x_{V}}\right)^{x_{P}}, d k=\operatorname{KDF}(v k),\left(p k^{\prime}, s k^{\prime}\right) \leftarrow \operatorname{Gen}\left(1^{k}\right), \\
& e=E_{d k}\left(p k^{\prime}\right), t=\operatorname{Sign}_{s k^{\prime}}(M), \text { where } x_{P}, x_{V} \text { denotes the secret keys of the prover }
\end{aligned}
$$
$P U$ and the verifier $V U$ respectively.

Finally, the prover $P U$ sends the authenticator Authen $=(e, t)$ and the message $M$ to the verifier $V U$.

(4) V: Having received the authenticator Authen $=(e, t)$ and the message $M$, the verifier $V U$ proceeds as follows: 


$$
\begin{aligned}
& v k=\left(g^{x_{P}}\right)^{x_{V}}, d k=K D F(v k), p k=D_{d k}(e) \\
& \text { If } p k=\perp \text { or } \operatorname{Vrfy}_{p k}(M, t) \neq 1 \text {, then outputs } 0 \text {. Otherwise outputs } 1 \text { to accept the }
\end{aligned}
$$

signature. The correctness of our scheme is obvious.

(5) Sim: Given the public key $g^{x_{P}}$ of the prover, it is obvious that the verifier is able to simulate the identically distributed authenticators by computing the trapdoor $\left(g^{x_{P}}\right)^{x_{V}}$.

\section{Security Analysis}

Theorem 1: Assume that (1) DDH assumption hold over $G$ with prime order $q$; (2) $K D F$ is a secure key derivation function; (3) $S K E=(E, D)$ is a INT-PTXT secure symmetric encryption scheme; (4) (Gen, Sign, Vrfy) is a signature scheme secure under one-time chosen message attack. Then our non-interactive deniable authentication scheme is unforgeable.

Proof: We define a game called Game 0 between an adversary $A$ and a simulator $S$. At first, the instructions in $\operatorname{Init}\left(1^{k}\right)$ is executed by $S$.

\section{Game 0}

$\operatorname{Init}\left(1^{k}\right)$

Begin

I0: The simulator $S$ generates the public parameter $\langle G, g, q\rangle$ and picks a key derivation function $K D F$, a one-time secure signature scheme (Gen, Sign, Vrfy) and a symmetric encryption scheme $S K E=(E, D)$.

I1: $S$ picks $x_{P}, x_{V} \leftarrow_{R} Z_{q}$ and computes $p k_{P}=g^{x_{P}}, p k_{V}=g^{x_{V}}$;

I2: $S$ computes $v k=\left(g^{x_{V}}\right)^{x_{P}}, d k=K D F(v k), i \leftarrow 0$;

I3: $S$ provides $A$ with $\langle G, g, q\rangle$, the description of $K D F$, (Gen, Sign, Vrfy) , $S K E=(E, D)$ and the public keys $p k_{P}, p k_{V}$.

End 
Assume without loss of generality that $A$ makes exactly $Q$ Conv queries. For i-th Conv query, we denote the intermediate values produced by the simulator by $\left(p k_{i}^{\prime}, s k_{i}^{\prime}\right), e_{i}, t_{i}$. When processing each Conv query $M$ issued by $A, S$ invokes the procedure $\operatorname{Conv}(M)$ and returns the output to $A$.

\section{$\operatorname{Conv}(M)$}

Begin

C1: $i \leftarrow i+1, M_{i} \leftarrow M$, generates $\left(p k_{i}^{\prime}, s k_{i}^{\prime}\right) \leftarrow \operatorname{Gen}(\cdot)$;

C2: computes $e_{i}=E_{d k}\left(p k_{i}^{\prime}\right), t_{i}=\operatorname{Sign}_{s k_{i}^{\prime}}\left(M_{i}\right)$ and returns Authen ${ }_{i}=\left(e_{i}, t_{i}\right) ;$

End

Finally, the adversary $A$ outputs $M^{*},\left(e^{*}, t^{*}\right)$. Then the simulator $S$ computes $p k^{*}=D_{d k}\left(e^{*}\right)$. If $p k^{*} \neq \perp$ and $\operatorname{Vrfy}_{p k^{*}}\left(M^{*}, t^{*}\right)=1, A$ wins Game 0.

Game 0 is exactly the $\operatorname{Exp}_{\mathrm{NDI}, \mathrm{A}}^{\mathrm{imp}}(k)$ game used to define unforgeability of non-interactive deniable authentication schemes. Then we prove theorem 1 by using a sequence of games. We define $X_{i}$ to be the event that $A$ wins in Game $i$.

\section{Game 1}

Game 1 is the same as Game 0 except that line I2 in the procedure Init is modified as follows:

$\operatorname{Init}\left(1^{k}\right)$

Begin

I0: Unchanged;

I1: Unchanged;

I2: $v k \leftarrow{ }_{R} G ; d k=K D F(v k) ; i \leftarrow 0$

I3: Unchanged.

End

Lemma 1: There exists an efficient adversary $A_{1}$ such that

$$
\left|\operatorname{Pr}\left[X_{0}\right]-\operatorname{Pr}\left[X_{1}\right]\right| \leq A d v_{A_{1}, G}^{D D H}(k) .
$$

Proof: $A_{1}$ takes the description of $\langle G, q\rangle$ and $\tau=\left(g_{1}, g_{2}, u_{1}, u_{2}\right)$ as input. Then $A_{1}$ 
runs a hybrid game with the adversary $A$ according to the instructions of the simulator in Game 0 except that the procedure Init is modified as follows:

$\operatorname{Init}\left(1^{k}\right)$

Begin

I0: $A_{1}$ sets $g \leftarrow g_{1}$ and picks $K D F$, (Gen, Sign, $\left.\operatorname{Vrfy}\right)$ and $S K E=(E, D)$.

I1: $A_{1}$ sets $p k_{P}=g_{2}, p k_{V}=u_{1}$;

$\mathrm{I} 2: v k \leftarrow u_{2} ; d k=K D F(v k), \quad i \leftarrow 0 ;$

I3: $A_{1}$ provides $A$ with $\langle G, g, q\rangle$, the description of $K D F$, (Gen, Sign,Vrfy) , $S K E=(E, D)$ and the public keys $p k_{P}, p k_{V}$.

End

$A_{1}$ outputs 1 if $A$ wins and outputs 0 otherwise. If $\tau$ is a DH tuple, the hybrid game acts like Game 0 , and if $\tau$ is a random tuple, the hybrid game acts like Game 1 . Let $\varepsilon=\left|\operatorname{Pr}\left[X_{0}\right]-\operatorname{Pr}\left[X_{1}\right]\right|$. The distinguishing advantage of $A_{1}$ is at least $\varepsilon$.

\section{Game 2}

Game 2 is the same as Game 1 except that line I2 in the procedure Init is modified as follows:

$\operatorname{Init}\left(1^{k}\right)$

Begin

I0: Unchanged;

I1: Unchanged;

I2: $v k \leftarrow{ }_{R} G ; d k \leftarrow\{0,1\}^{k}, i \leftarrow 0 ;$

I3: Unchanged.

End

Lemma 2: There exists an efficient adversary $A_{2}$ such that

$$
\left|\operatorname{Pr}\left[X_{1}\right]-\operatorname{Pr}\left[X_{2}\right]\right| \leq A d v_{A_{2}}^{K D F}(k)
$$

Proof: Observe that $v k$ in Game 1 is chosen at random and used only once as input to $K D F$. Thus the lemma follows from the security definition of KDF functions. 
Assume that the adversary $A$ outputs $M^{*},\left(e^{*}, t^{*}\right)$ in Game 2. At this point, let Reuse be the event that $D_{d k}\left(e^{*}\right)=p k_{i}^{\prime}$ for some $1 \leq i \leq Q$. Obviously we have

$$
\operatorname{Pr}\left[X_{2}\right]=\operatorname{Pr}\left[X_{2} \wedge \text { Reuse }\right]+\operatorname{Pr}\left[X_{2} \wedge \overline{\text { Reuse }}\right]
$$

Lemma 3: There exists an efficient adversary $A_{3}$ such that

$$
\operatorname{Pr}\left[X_{2} \mid \text { Reuse }\right] \cdot \frac{1}{Q} \leq A d v_{A_{3}}^{1 C M A}(k)
$$

Proof: At first, the challenger in the experiment $\operatorname{Exp}_{\text {sig }}^{1 C M A}(k)$ generates $(p k, s k) \leftarrow \operatorname{Gen}(\cdot)$. Then the adversary $A_{3}$ takes the description of one-time secure signature scheme (Gen, Sign, Vrfy) and the public key $p k$ as input and simulates the environment of Game 2 as follows:

$\operatorname{Init}\left(1^{k}\right)$

Begin

I0: $A_{3}$ generates the public parameter $\langle G, g, q\rangle$ and picks $K D F$ and $S K E=(E, D)$.

I1: $A_{3}$ picks $x_{P}, x_{V} \leftarrow{ }_{R} Z_{q}$ and computes $p k_{P}=g^{x_{P}}, p k_{V}=g^{x_{V}}$;

I2: $d k \leftarrow\{0,1\}^{k} ; j \leftarrow{ }_{R}\{1, \cdots, Q\}, i \leftarrow 0 ;$

I3: $A_{3}$ provides $A$ with $\langle G, g, q\rangle$, the description of KDF, (Gen, Sign,Vrfy) , $S K E=(E, D)$ and the public keys $p k_{P}, p k_{V}$.

End

When processing each Conv query $M$ issued by $A, A_{3}$ invokes $\operatorname{Conv}(M)$ and returns the output to $A$.

\section{$\operatorname{Conv}(M)$}

Begin

$$
i \leftarrow i+1, M_{i} \leftarrow M
$$


If $i \neq j$ then

$$
\begin{aligned}
& \left(p k_{i}^{\prime}, s k_{i}^{\prime}\right) \leftarrow \operatorname{Gen}(\cdot) ; \\
& e_{i}=E_{d k}\left(p k_{i}^{\prime}\right), t_{i}=\operatorname{Sign}_{s k_{i}^{\prime}}\left(M_{i}\right) \text { and return } \text { Authen }_{i}=\left(e_{i}, t_{i}\right) ;
\end{aligned}
$$

Else

$e_{i}=E_{d k}(p k), t_{i}=O\left(M_{i}\right)$ and return Authen $_{i}=\left(e_{i}, t_{i}\right)$, where $O$ denotes the signing oracle which can be accessed by $A_{3}$ only once in the experiment $\operatorname{Exp}_{\text {sig }}^{1 C M A}(k)$.

End

After the adversary $A$ outputs $M^{*},\left(e^{*}, t^{*}\right), A_{3}$ decrypts $e^{*}$ to obtain $p k^{*}=D_{d k}\left(e^{*}\right)$. If $p k^{*} \neq p k$, then $A_{3}$ aborts. Otherwise, $A_{3}$ outputs $\left(M^{*}, t^{*}\right)$ if $A$ wins.

If $A_{3}$ correctly guess the index $j$ when the event Reuse happens, we have $\operatorname{Pr}\left[X_{2} \mid\right.$ Reuse $] \cdot \frac{1}{Q} \leq A d v_{A_{3}}^{1 C M A}(k)$.

Lemma 4: There exists an efficient adversary $A_{4}$ such that

$$
\operatorname{Pr}\left[X_{2} \mid \overline{\text { Reuse }}\right] \leq A d v_{A_{4}}^{P T X T}(k) .
$$

Proof: At first, the challenger in the experiment $\operatorname{Exp}_{S E}^{P T X T}(k)$ generates $d k \leftarrow\{0,1\}^{k}$. Then the adversary $A_{4}$ takes the description of $\operatorname{SKE}=(E, D)$ as input and simulates the environment of Game 2 as follows:

$\operatorname{Init}\left(1^{k}\right)$

Begin

I0: $A_{4}$ generates the public parameter $\langle G, g, q\rangle$ and picks $K D F$, (Gen, Sign, Vrfy).

I1: $A_{4}$ picks $x_{P}, x_{V} \leftarrow_{R} Z_{q}$ and computes $p k_{P}=g^{x_{P}}, p k_{V}=g^{x_{V}}$;

I2: $i \leftarrow 0$ 
I3: $A_{4}$ provides $A$ with $\langle G, g, q\rangle$, the description of $K D F$, (Gen, Sign,Vrfy) , $S K E=(E, D)$ and the public keys $p k_{P}, p k_{V}$.

End

When processing each Conv query $M$ issued by $A, A_{4}$ invokes $\operatorname{Conv}(M)$ and returns the output to $A$.

$\operatorname{Conv}(M)$

Begin

$\mathrm{C} 1: \quad i \leftarrow i+1, M_{i} \leftarrow M ;\left(p k_{i}^{\prime}, s k_{i}^{\prime}\right) \leftarrow \operatorname{Gen}(\cdot) ;$

C2: $e_{i}=O\left(p k_{i}^{\prime}\right), t_{i}=\operatorname{Sign}_{\text {sk }_{i}^{\prime}}\left(M_{i}\right)$ and return Authen $_{i}=\left(e_{i}, t_{i}\right)$, where $O$ denotes the encryption oracle accessed by $A_{4}$ in the experiment $\operatorname{Exp}_{S E}^{P T X T}(k)$.

End

After the adversary $A$ outputs $M^{*},\left(e^{*}, t^{*}\right), A_{4}$ outputs $e^{*}$. The challenger in the experiment $\operatorname{Exp}_{S E}^{P T X T}(k)$ decrypts $e^{*}$ to obtain $p k^{*}=D_{d k}\left(e^{*}\right)$. Obviously, when the event $\overline{\text { Reuse }}$ happens, $A_{4}$ wins if $A$ wins in Game 2.

As $A_{4}$ perfectly simulates the environment of Game 2 for the adversary $A$, so we have $\operatorname{Pr}\left[X_{2} \mid \overline{\text { Reuse }}\right] \leq A d v_{A_{4}}^{P T X T}(k)$. By combining the above results, we have:

$$
\operatorname{Adv}_{\mathrm{NDI}, \mathrm{A}}^{\mathrm{imp}}(k) \leq A d v_{A_{1}, G}^{D D H}(k)+A d v_{A_{2}}^{K D F}(k)+\max \left(Q \cdot A d v_{A_{3}}^{1 C M A}(k), A d v_{A_{4}}^{P T X T}(k)\right)
$$

By assumption, the right-hand side of the above equation is negligible, which finishes the proof.

\section{Performance Analysis}

In this section, we evaluate the performance of our construction and other related non-interactive deniable authentication schemes with provable security $[22,24]$ in terms of the computational cost. The result is stated in Table 1. Exp denotes an exponentiation operation, which is the most time-consuming operation used in these schemes. For ease of comparison, 
we use the signature scheme in [12] which is one-time secure in the standard model to instantiate our construction. Note that the computational cost of a prover in our scheme should take the key generation of one-time signature scheme into consideration.

In the table, the computational cost of a multi-exponentiation (that is, computing $g_{1}{ }^{a} g_{2}^{b}$ or $g_{1}{ }^{a} g_{2}^{b} g_{3}{ }^{c}$ ) is assumed to be at most 1.5 exponentiations[18]. Although the scheme [24] is more efficient than others, the efficiency of our construction can be further reduced when the key pair of one-time signature scheme can be pre-computed and stored such that only one exponentiation is needed to compute the shared secret $v k=\left(g^{x_{V}}\right)^{x_{P}}$. Such pre-computation technique does not apply to the schemes in [22,24]. Moreover, our scheme is proven to be secure in the standard model which provides stronger security guarantee than the random oracle model.

Table 1. Performance comparison

\begin{tabular}{|c|c|c|c|}
\hline Scheme & $\begin{array}{c}\text { Prover's } \\
\text { computational cost }\end{array}$ & $\begin{array}{c}\text { Verifier's } \\
\text { computational cost }\end{array}$ & $\begin{array}{c}\text { Setup } \\
\text { assumptions }\end{array}$ \\
\hline $\begin{array}{c}\text { Wang et al's } \\
\text { Scheme [22] }\end{array}$ & $3.5 \mathrm{Exp}$ & $4.5 \mathrm{Exp}$ & $\begin{array}{c}\text { The random } \\
\text { oracle model }\end{array}$ \\
\hline $\begin{array}{c}\text { Youn et al's } \\
\text { Scheme [24] }\end{array}$ & $2 \mathrm{Exp}$ & $2.5 \mathrm{Exp}$ & $\begin{array}{c}\text { The random } \\
\text { oracle model }\end{array}$ \\
\hline $\begin{array}{c}\text { The proposed } \\
\text { Scheme }\end{array}$ & $4.5 \mathrm{Exp}$ & $2.5 \mathrm{Exp}$ & $\begin{array}{c}\text { The standard } \\
\text { model }\end{array}$ \\
\hline
\end{tabular}

\section{Conclusion}

In this paper, we provide a generic construction for deniable authentication schemes that can be instantiated without bilinear groups. Deniability of our scheme is achieved by the property of the Diffie-Hellman key exchange protocol. In the following, we prove our scheme to be unforgeable in the standard model by sequences of games. In the process of proof, we make use of the notion of integrity of plaintexts with regard to symmetric encryption. Finally we show that the computational cost of our construction can be dramatically reduced by applying pre-computation technique such that the performance of our construction is 
comparable to the most efficient non-interactive deniable authentication scheme [24] whose security is based on the random oracle model.

\section{Acknowledgement}

This work is supported by Natural Science Foundation of Higher Education Institutions, in Jiangsu Province office of education, P.R. China (Grant No. 10KJD520005), National Natural Science Foundation of China(Grant No. 60803122), Innovative Foundation of Yangzhou University (Grant No. 2011CXJ022, 2011CXJ023).

\section{References}

[1] Y. Aumann, M.O. Rabin, “Authentication, enhanced security and error correcting codes”, in Proceedings of CRYPTO 1998. Springer, LNCS 1462, 1998, pp.299-303

[2] M. Bellare, M. Boldyreva, and A. Palacio, "An uninstantiable random oracle model scheme for a hybrid-encryption problem” EuroCrypt 2004, Springer, LNCS 3027, 2004, pp.171-188

[3] M. Bellare, R. Canetti and H. Krawczyk, "A Modular Approach to the Design and Analysis of Authentication and Key Exchange Protocols”, proc. of 30th Symposium on Theory of Computing (STOC), ACM, 1998, pp. 419-428

[4] M. Bellare , C. Namprempre, “Authenticated Encryption: Relations among Notions and Analysis of the Generic Composition paradigm”, Journal of Cryptology, 21(4)(2008), 469-491

[5] M. Bellare, C. Namprempre , G. Neven, “Security Proofs for Identity-Based Identification and Signature Schemes”, Journal of Cryptology, 22(1)(2009), pp.1-61

[6] A. Bender, J. Katz, R. Morselli, “Ring Signatures: Stronger Definitions, and Constructions without Random oracles”, Journal of Cryptology, 22(1)(2009), pp.114-138

[7] R. Canetti, O. Goldreich and S. Halevi, “The random oracle methodology, revisited”. In STOC, 1998, pp. 209-218

[8] S.S.M. Chow, V.K.-W.Wei, J.K. Liu, T.H. Yuen, "Ring signatures without random oracles”, in Proc. ACM Symposium on Information, Computer and Communications Security (ASIACCS) 2006 (ACM, New York, 2006), pp. 297-302 
[9] X. Deng, C. Lee, H. Lee, H. Zhu, “Deniable Authentication Protocols”, IEE Proc.Comput. Digit. Tech., 148(2)(2001), pp.101-104

[10] C. Dwork, M. Naor, A. Sahai, “Concurrent zero-knowledge”, in: Proceedings of 30th ACM STOC’98, 1998, pp.409-418

[11] L. Fan, C.X. Xu, J.H. Li, "Deniable authentication protocol based on Diffie-Hellman algorithm”, Electronics Letters, 38 (4)(2002), pp.705-706

[12] J. Groth, "Simulation-sound NIZK proofs for a practical language and constant size group signatures", in ASIACRYPT’2006, 2006, pp.339-358

[13] M. Jacobsson, K. Sako, R, Impagliazzo, "Designated verifier proofs and their applications", in EUROCRYPT’1996, LNCS 1070, 1996, pp.143-154

[14] W.B. Lee, C.C. Wu, W.J. Tsaur, "A novel deniable authentication protocol using generalized ElGamal signature scheme”, Information Sciences, 177(2007), pp.1376-1381

[15] R. Lu, Z.F. Cao, “A new deniable authentication protocol from bilinear pairings”, Applied Mathematics and Computation, 168(2005), pp.954-961.

[16] R. Lu, Z.F. Cao, "Non-interactive deniable authentication protocol based on factoring”, Computer Standards \& Interfaces, 27 (2005), pp.401-405.

[17] R. Lu, Z.F. Cao, "Erratum to "Non-interactive deniable authentication protocol based on factoring”’, Computer Standards \& Interfaces, 29 (2007), pp.275.

[18] A.J. Menezes, P.C.van Oorschot and S.A. Vanstone, "Handbook of Applied Cryptography”, CRC Press, 1997

[19] M.D. Raimondo and R. Gennaro, “New Approaches for Deniable Authentication”, Journal of Cryptology, 22(2009), pp.572-615

[20] Z. Shao, "Efficient deniable authentication protocol based on generalized ElGamal signature scheme”, Computer Standards \& Interfaces, 26 (2004), pp.449-454.

[21] W. Susilo and Y. Mu, “Non-interactive Deniable Ring Authentication”, in ICISC’2003, LNCS 2971,2003, pp. 386-401

[22]Bin Wang, ZhaoXia Song, “A non-interactive deniable authentication scheme based on designated verifier proofs” . Information Science, 179(6)(2009), pp.858-865

[23] E.J. Yoon, E. K. Ryu, K.Y. Yoo, “Improvement of Fan et al.’s deniable authentication protocol based on Diffie-Hellman algorithm”, Applied Mathematics and Computation, 167 
(2005), pp.274-280

[24]T.Y Youn, C.Lee, Y.H., Park, “An efficient non-interactive deniable authentication scheme based on trapdoor commitment schemes”, Computer Communications, 34(2011), pp.353-357

[25] R.W. Zhu, D.S. Wong, and C.H. Lee, “Cryptanalysis of a suite of deniable authentication protocols”, IEEE Communications Letters, 10(6) (2006), pp.504-506 